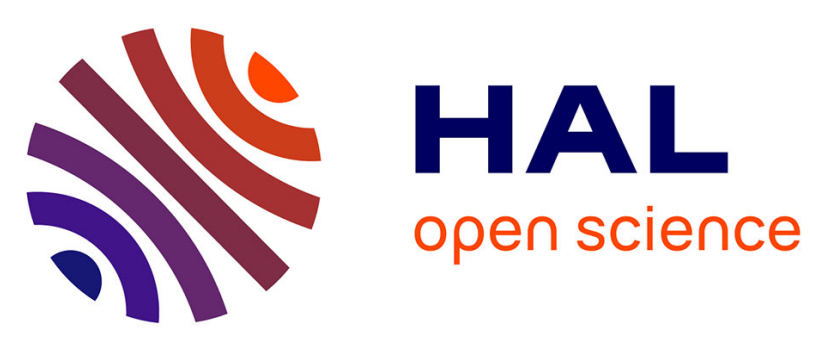

\title{
3D Analytical Computation of the Torque in Axial Flux Permanent Magnets Couplings Based on Charges Model and Images Method
}

Mahammed Messadi, Larbi Hadjout, Youcef Ouazir, Noureddine Takorabet, Thierry Lubin, Smail Mezani

\section{To cite this version:}

Mahammed Messadi, Larbi Hadjout, Youcef Ouazir, Noureddine Takorabet, Thierry Lubin, et al.. 3D Analytical Computation of the Torque in Axial Flux Permanent Magnets Couplings Based on Charges Model and Images Method. 3rd International Conference on Electrical Sciences and Technologies in Maghreb (CISTEM), Oct 2018, Alger, Algeria. pp.529-534. hal-01910626

\section{HAL Id: hal-01910626 \\ https://hal.science/hal-01910626}

Submitted on 1 Nov 2018

HAL is a multi-disciplinary open access archive for the deposit and dissemination of scientific research documents, whether they are published or not. The documents may come from teaching and research institutions in France or abroad, or from public or private research centers.
L'archive ouverte pluridisciplinaire HAL, est destinée au dépôt et à la diffusion de documents scientifiques de niveau recherche, publiés ou non, émanant des établissements d'enseignement et de recherche français ou étrangers, des laboratoires publics ou privés. 


\section{D Analytical Computation of the Torque in Axial Flux Permanent Magnets Couplings Based on Charges Model and Images Method}

\author{
M. Messadi ${ }^{1}$, L. Hadjout $^{1}$, Y.Ouazir $^{1}$ \\ ${ }^{1}$ LSEI-Université des Sciences et Technologie Houari \\ Boumediene, BP N³2, 16111, Alger, Algérie, \\ messadi_m@yahoo.fr
}

\begin{abstract}
This paper presents a simple and accurate 3D analytical expressions to compute the force and torque of an axial flux magnetic couplings (AFMC) based on the equivalent magnetic charges and the images method. The proposed model is formulated and solved in a 3D Cartesian coordinate system by considering the assumption of linearization around the mean radius. Firstly, the magnetic flux density due to the cubic permanent magnets (PM), of one side of the magnetic coupling, is computed in 3D considering the magnetic end effects where the iron yokes influence is considered thanks to the images method. Secondly, the force and the torque among the magnets located in the opposite sides are obtained using the analogy between the electrostatic and magnetostatic forces. The derived analytical expressions depend directly on the geometrical and physical parameters of the AFMC. The analytical results are compared to those obtained with finite element simulation and experimental measurements.
\end{abstract}

Keywords-magnetic coupling, permanent magnets, charges model, method of images, Electrostatic-Magnetostatic Analogy, three-dimensional (3-D) analytical model.

\section{INTRODUCTION}

Magnetic couplings are actuators that transmit a torque without mechanical contact. Therefore, they are well suited for applications requiring mechanical isolation between two moving parts. The air gap may contain a wall that separates two different media such as in toxic or corrosive environments [1]. Magnetic couplings can have linear or rotary topologies, with axial or radial flux.

The AFMC have a strong magnetic end effects that need to be taken into account in the modeling [2]-[4]. In the literature many modeling methods for magnetic couplings, using analytical [5], [6] or numerical models [7], [8] are presented. The use of a full three dimensional finite element model (3D FEM) is obviously one of the most suitable way for the modeling of AFMC. However, this method is time-consuming and needs a huge space memory [7], [8].

Analytical models for magnetic couplings are usually based on 2-D approximations. This model does not allow taking into account the edge effects, which can induce errors of up to $30 \%$ on the prediction of global quantities [2], [9]. To improve the accuracy of the $2 \mathrm{D}$ models, a multi-layer approach is used for axial flux PMs machines, by making several 2D calculations for different radius [4], [11]. This quasi-3D calculation improves the results, but the resolution times are more important [19].

\author{
N. Takorabet ${ }^{2}$, T. Lubin $^{2}$, S. Mezani ${ }^{2}$ \\ ${ }^{2}$ GREEN-Université de Lorraine, Faculté des Sciences et \\ Technologies, BP 239, 54506, Vandœuvre-lès-Nancy, \\ France
}

3D analytical models found in the literature are essentially based on integral methods [12], [13]. These models allow a 3D calculation of the magnetic field in vacuum for simple geometries, using the principle of equivalent sources. In the case of cubic PM the force and the interaction torque are calculated in vacuum by the use of the equivalent magnetic charge model [14], [16].

More recently, 3D analytical models, based on the resolution of the Maxwell equations in the Cartesian coordinates, are developed. For this purpose, the cylindrical topology is linearized into a 3D linear topology extended to infinity, neglecting the curvature effect [3], [17]. These methods do not allow to take into account the effects of extremities due to the finite length of the linear actuators.

In this paper, the equivalent magnetic charges model and the image method are combined to develop a new 3D analytical computation of the interaction force and torque exerted between cubic PM supported by ferromagnetic yokes. The proposed model is used to analyze the force and torque of permanent magnets AFMC. In order to show the accuracy of the analytical model the results are compared with those obtained by 3D FEM and experimental measurements.

\section{Studied DeVICE AND Assumptions}

The studied magnetic coupling is represented on Fig. 1. It is composed of two inductors with sector-shaped permanent magnets supported by iron yokes. The inductors are based on rare-earth permanent magnets axially magnetized and arranged to obtain alternatively north and south poles. The parameters of the studied device are given in Table. I.

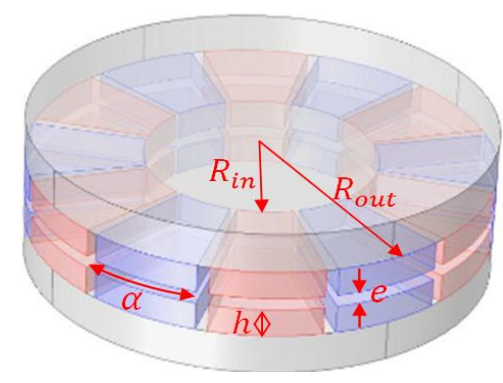

(a)

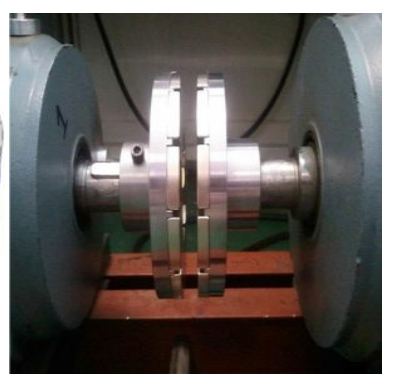

(b)
Fig.1. (a) Geometry of the studied AFMC. (b) prototype 
TABLE I: PARAMETERS OF THE STUDIED AFMC [2]

\begin{tabular}{|l|l|l|}
\hline Symbol & Quantity & value \\
\hline Rout & Outer radius of the magnets & $60 \mathrm{~mm}$ \\
\hline Rin & Inner radius of the magnets & $30 \mathrm{~mm}$ \\
\hline$h$ & Magnets thickness & $7 \mathrm{~mm}$ \\
\hline $\mathrm{e}$ & Thickness of the air gap & variable \\
\hline$\alpha$ & PMs pole-arc to pole-pitch ratio & 0.9 \\
\hline$p$ & Pole-pairs number & 6 \\
\hline$B r$ & Residual induction of permanent magnets & $1.25 \mathrm{~T}$ \\
\hline
\end{tabular}

To simplify the analysis and overcome the problems encountered using the cylindrical coordinates [17], a linearization assumption at the mean radius is used. Then the problem can be solved in 3-D Cartesian coordinate. So, curvature effect is neglected.

With this assumption, the 3D cylindrical geometry is replaced by a linear one extended to infinity in both directions along the $\mathrm{y}$ axis, where the coordinates $\mathrm{x}, \mathrm{y}$ and $\mathrm{z}$ represent respectively the radial direction, the circumferential direction and the axial direction. Fig. 2 illustrates this assumption under one pole of the permanent magnet inductor.

After linearization, the dimensions of the equivalent linearized AFMC are given by:

$$
2 b=R_{\text {out }}-R_{\text {in }}, \quad 2 a=\alpha \tau_{p}
$$

With $\quad \tau_{p}=\frac{\pi}{p} R_{\text {mean }} \quad$ and $\quad R_{\text {mean }}=\left(R_{\text {out }}+R_{\text {in }}\right) / 2$

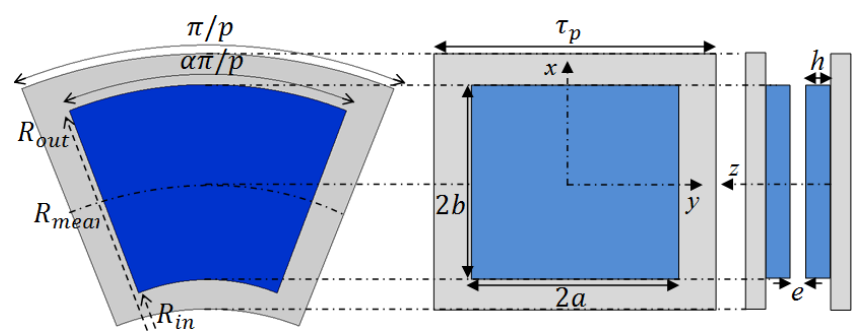

Fig.2. Linearization at the mean radius of the AFMC geometry.

In next section the proposed modeling method of the studied AFMC is presented. The following assumptions are adopted.

- The PM is considered with a rigid and uniform magnetization $\vec{M}$.

- The relative permeability is considered infinite for the ferromagnetic parts and is equal to 1 for the PMs.

\section{COMPUTATION METHOD}

The calculation of the force and torque is carried out in two steps:

- We consider only the magnets on one side of the magnetic coupling, the magnetic flux density is calculated using the equivalent charges model, and the iron yokes influence is considered thanks to the images method.
- The force and the torque acting on the magnets placed on the opposite side are calculated, using the law of the Lorentz force, obtained by analogy with the electrostatic force.

\section{A. Magnetic Flux density}

The developed method consists in calculating the magnetic field produced by each rectangular shaped magnet separately. Using the Coulombian model, each magnet is replaced by two equivalent magnetic surface charge density $+\sigma$ and $-\sigma$, located on its upper and lower faces (Fig. 3). For a magnet with a rigid and uniform magnetization in the $\mathrm{z}$-direction $\vec{M}$, the equivalent surface charge density is given by, [15], [16]:

$\sigma=\vec{M} \cdot \vec{n}$

where $\vec{n}$ is the normal vector of the PM surfaces.

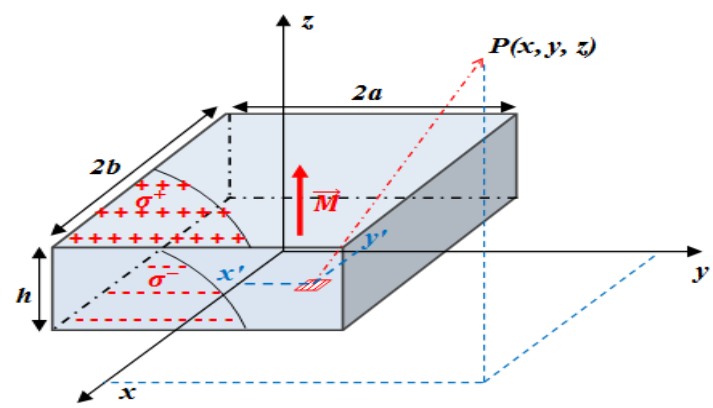

Fig.3. Coulombian model of Permanent Magnet

In vacuum, the magnetic flux density $\vec{B}(p)$ of arbitrary position $p(x, y, z)$ in space due to the magnetic charge surface is calculated using Coulombian low given by, [18]:

$\vec{B}(p)=\frac{1}{4 \pi} \iint_{S} \sigma \frac{\vec{r}}{r^{3}} d s$

where, $\vec{r}$ is the distance between the source and the local point $p(x, y, z)$.

The magnetic flux density at any point in the space, for one PM block is obtained by calculating the surface integral, of the equivalent magnetic charge, over the top and bottom surfaces of the magnet.

To take into account the boundary conditions at the interfaces between the air gap and the ferromagnetic yokes, the images method is used [10], [15], [18]. The principle consists replace the effects of a ferromagnetic plane by equivalent magnetic charges behind the plane; the desired field is then obtained by the superposition of the applied charges and its images.

For equivalent magnetic charges density of the magnet located between two ferromagnetic yokes, the image's theory consists in replacing the two planes by infinity of images with alternatively negative and positive signs, (Fig. 4). Moreover, the lower sheet of the magnet disappears completely because it merges with the image of the adjacent ferromagnetic medium. 


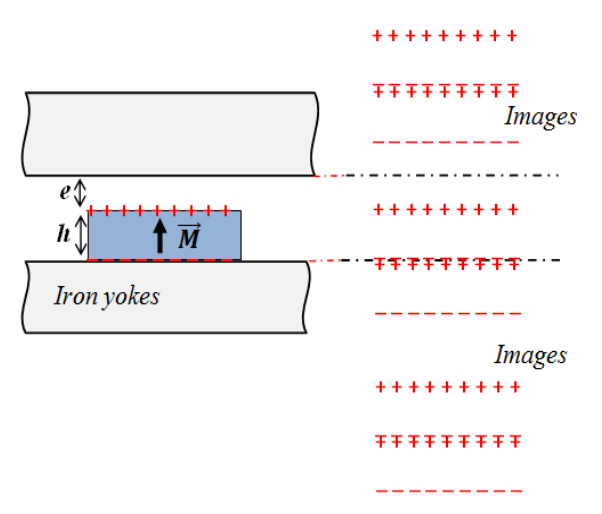

Fig.4. Principle of the images method.

Applying this approach to the studied AFMC (Fig. 5), the expression of the magnetic flux density for one bloc magnet, is determined by superposition of all image contributions and the original magnets. The air gap magnetic flux density is obtained by superposition of magnetic field produced by all magnet blocs.

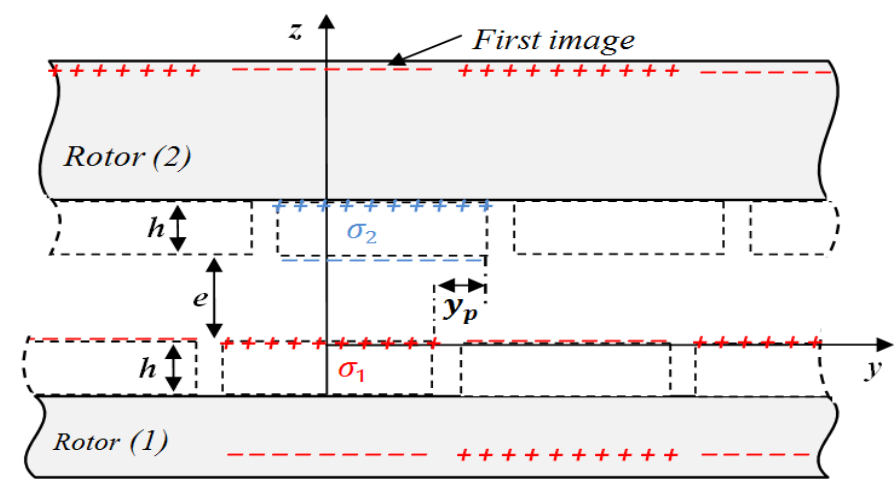

Fig.5. Equivalent charges model of the AFMC in Cartesian coordinates axial

Starting from the solution given by (3), and considering only the magnets on one inductor of the coupling, The resulting magnetic flux density is given by :

$$
\left\{\begin{array}{l}
B_{x}=\frac{\sigma_{1}}{4 \pi} \sum_{k=1}^{2 p} \sum_{i=0}^{1} \sum_{j=0}^{1}-(-1)^{i+j+k} \\
\times\left[\sum_{m=0}^{+\infty}(-1)^{m} \ln \left(Y+R_{1}\right)+\sum_{m=1}^{+\infty}(-1)^{m} \ln \left(Y+R_{2}\right)\right] \\
B_{y}=\frac{\sigma_{1}}{4 \pi} \sum_{k=1}^{2 p} \sum_{i=0}^{1} \sum_{j=0}^{1}-(-1)^{i+j+k} \\
\times\left[\sum_{m=0}^{+\infty}(-1)^{m} \operatorname{Ln}\left(X+R_{1}\right)+\sum_{m=1}^{+\infty}(-1)^{m} \operatorname{Ln}\left(X+R_{2}\right)\right] \\
B_{z}=\frac{\sigma_{1}}{4 \pi} \sum_{k=1}^{2 p} \sum_{i=0}^{1} \sum_{j=0}^{1}(-1)^{i+j+k} \\
\times\left[\sum_{m=0}^{+\infty}(-1)^{m} \operatorname{tg}^{-1}\left(\frac{Y X}{Z_{1} R_{1}}\right)+\sum_{m=1}^{+\infty}(-1)^{m} \operatorname{tg}^{-1}\left(\frac{Y X}{Z_{2} R_{2}}\right)\right]
\end{array}\right.
$$

where for a point $p(x, y, z)$ :

$$
\begin{aligned}
& X=\left(\mathrm{x}-(-1)^{j} b\right) ; Y=\left(\mathrm{y}-(\mathrm{k}-1) \tau_{p}-(-1)^{i} a\right) ; \\
& R_{1}=\sqrt{X^{2}+Y^{2}+Z_{1}^{2}} ; R_{2}=\sqrt{X^{2}+Y^{2}+Z_{2}^{2}} .
\end{aligned}
$$

We can distinguish four sets of PM's images. Their heights are:

$$
\begin{aligned}
& Z_{1}=\left\{\begin{array}{cl}
z+m(2 h+e) & \text { m even } \\
z+(m+1) h+(m+1)(e+h) & \text { modd } \\
\text { meven }
\end{array}\right. \\
& Z_{2}=\left\{\begin{array}{cl}
z-m(2 h+e) & \text { modd } \\
z-(m-1) h-(m+1)(e+h)
\end{array}\right.
\end{aligned}
$$

$a, b$ and $h$ are the magnet dimensions as shown in Fig. 2 and $m$ is the number of the PM image which must be enough to have a stable solution

\section{B. Forces Expressions}

The interaction forces between the two inductors of the AFMC are obtained using the Electrostatic-Magnetostatic analogy, [3]. The Lorentz force exerted on the uniform electric surface charge $\sigma_{s}$ subjected to an electric field $\vec{E}$, is:

$\vec{F}=\sigma_{s} \iint_{s} \vec{E} d s$

Where, $s$ is the surface which carries $\sigma_{s}$.

By analogy to the electrosatic force (5), the magnetic force exerted on magnetic charge surface $\sigma_{s}$ subjected to magnetic flux density $\vec{B}$, is then:

$$
\vec{F}=\sigma_{s} \iint_{s} \vec{B} d s
$$

From (6), we can show that to calculate the interaction forces between the two inductors of the AFMC, all we need is to determine the magnetic field produced by the first inductor and replace the magnets of the second by their equivalent magnetic charge model. So the force will be obtained using (6), where the integration of the magnetic flux $\vec{B}$ is performed on the surfaces carrying $+\sigma_{2}$ and $-\sigma_{2}$, equivalent to second inductor magnets, (Fig. 5).

The use of the Lorentz force, for a system with ferromagnetic yokes, gives a good values only for the tangential forces $\left(F_{x}\right.$ and $\left.F_{y}\right)$, because there is no other material than air in these two directions [3]. However, as the ferromagnetic materials are present in the $z$ direction, this method does not give the good value of the axial force (iron is not considered for the magnets of the second inductor), and we have to use Maxwell stress tensor.

Furthermore, the relative permeability is considered infinite for the ferromagnetic parts $(\mu r \rightarrow \infty)$. The boundary condition on the iron-air interface is given by:

$$
\vec{n} \times \vec{H}=0
$$


According to this condition, the tangential components $H x$ and $H y$ of the magnetic field, will be null on the charged surface $+\sigma$ at $z=h+e$, (Fig. 5). Hence, the integation is only performed on the charged surface $-\sigma$ at $z=e$, (Fig. 5).

Moreover, taking into account the periodicity, the calculation is limited under one pole and then we multiply by the pole pair number $(2 p)$. After analytical integration, the obtained expressions of the force, components are:

$F_{x}\left(y_{p}\right)=2 p \frac{\sigma_{1} \sigma_{2}}{4 \pi} \sum_{k=1}^{2 p} \sum_{i=0}^{1} \sum_{j=0}^{1} \sum_{l=0}^{1} \sum_{n=0}^{1}-(-1)^{i+j+k+l+n}$
$\times\left[\sum_{m=0}^{+\infty}(-1)^{m} f_{x}\left(X, Y, Z_{1}, R_{1}\right)+\sum_{m=1}^{+\infty}(-1)^{m} f_{x}\left(X, Y, Z_{2}, R_{2}\right)\right]$

$F_{y}\left(y_{p}\right)=2 p \frac{\sigma_{1} \sigma_{2}}{4 \pi} \sum_{k=1}^{2 p} \sum_{i=0}^{1} \sum_{j=0}^{1} \sum_{l=0}^{1} \sum_{n=0}^{1}-(-1)^{i+j+k+l+n}$

$\times\left[\sum_{m=0}^{+\infty}(-1)^{m} f_{y}\left(X, Y, Z_{1}, R_{1}\right)+\sum_{m=1}^{+\infty}(-1)^{m} f_{y}\left(X, Y, Z_{2}, R_{2}\right)\right]$

where;

$f_{x}=\frac{\left(Y^{2}-Z^{2}\right)}{2} \ln (R-X)+X Y \ln (R-Y)+Y Z \tan ^{-1}\left(\frac{X Y}{Z R}\right)+\frac{X R}{2}$ $f_{y}=\frac{\left(X^{2}-Z^{2}\right)}{2} \ln (R-Y)+X Y \ln (R-X)+X Z \tan ^{-1}\left(\frac{X Y}{Z R}\right)+\frac{Y R}{2}$

with $\quad Y=\left((-1)^{n}-(-1)^{i}\right) a-(k-1) \tau_{p}+y_{p}$;

$X=\left((-1)^{l}-(-1)^{j}\right) b ;$

where $y_{p}$ represent the angular lag between the two inductors in Cartesian coordinates as shown in Fig. 5.

The axial magnetic force is obtained using the Maxwell stress tensor. The method consists in determining the magnetic flux density due to all magnets in each inductor separately by images method.

The axial force is derived from the integration of the Maxwell stress tensor on a surface located at the middle of the air gap versus the load angle. His expression is as follows:

$F_{z}\left(y_{p}\right)=\frac{2 p}{\mu_{0}} \iint_{S}^{\cdot}\left(\left(B_{z}\right)^{2}-B_{x}^{2}-B_{y}^{2}\right) d x d y$

with

$$
\begin{aligned}
& B_{x}=B_{x 1}(x, y, z)-B_{x 2}\left(x,\left(y+y_{p}\right), z\right) \\
& B_{y}=B_{y 1}(x, y, z)-B_{y 2}\left(x,\left(y+y_{p}\right), z\right) \\
& B_{z}=B_{z 1}(x, y, z)+B_{z 2}\left(x,\left(y+y_{p}\right), z\right)
\end{aligned}
$$

where, $\left(B_{x 1}, B_{y 1}, B_{z 1}\right)$ and $\left(B_{x 2}, B_{y 2}, B_{z 2}\right)$ are the magnetic flux density due to the first and the second inductor magnets, respectively.

\section{Torque Expression}

The Lorentz force method (6) is used in the proposed model to obtain the analytical torque expressions [14], [16]. Its general expression is given by:

$\vec{\Gamma}=\overrightarrow{\mathrm{r}} \times \overrightarrow{\mathrm{F}}=\sigma_{2} \iint_{S} \overrightarrow{\mathrm{r}} \times \overrightarrow{\mathrm{B}} \mathrm{ds}$

For, $\vec{r}=r_{x} \vec{\imath}+r_{y} \vec{\jmath}+r_{z} \vec{k}$, The torque can be also written as:

$\vec{\Gamma}=\sigma_{2} \iint_{s}\left(\begin{array}{c}r_{y} B_{z}-r_{z} B_{y} \\ r_{z} B_{x}-r_{x} B_{z} \\ r_{x} B_{y}-r_{y} B_{x}\end{array}\right) d s$

For the studied AFMC, the torque is calculated for a movement around the axis of rotation Oz. This axis has constant coordinates noted $\left(P_{x}, P_{y}\right)$ in the reference frame (OXYZ). So we are only interested in the $\mathrm{Z}$ component of the torque which is given by:

$\Gamma_{z}=\sigma_{2} \iint\left(\mathrm{x}-P_{x}\right) \cdot \mathrm{B}_{\mathrm{y}}-\left(\mathrm{y}-P_{y}\right) \cdot \mathrm{B}_{\mathrm{x}} d x d y$

Substituting the expression of the magnetic flux produced by the first inductor (4) into (13), after the analytical integrations, we found that the expression of the torque is directly linked to the force expressions as follows:

$\Gamma_{z}\left(y_{p}\right)=-P_{x} F_{y}-\left(-(k-1) \tau_{p}+y_{p}-P_{y}\right) F_{x}$

In a Cartesian coordinate the force $F_{x}$ is symmetrical along the $x$-direction and equal to zero $(F x=0)$. Hence, the corresponding torque also vanishes. For $P_{x}=R_{\text {mean }}$, the torque can be expressed as:

$\Gamma_{z}\left(y_{p}\right)=-R_{\text {mean }} F_{y}$

\section{RESULTS}

Using the proposed analytical model, a performance analysis of the studied AFMC is presented in this section.

In what follows, the accuracy of the analytical model is confirmed by a 3D FEM and experimental measurements performed on a prototype shown in Fig.1.b [2].

Fig. 6 and Fig. 7 show respectively, the normal magnetic flux densities distribution in the middle of the air-gap, along the circumferential direction ( $y$-direction) in the mean radius and along the radial direction ( $x$-direction) in the middle of a pole. These results are obtained under no-load condition for an air-gap value of $9.5 \mathrm{~mm}$.

It can be seen that the flux density waveforms predicted by the 3D developed model are in a good agreement compared to the experiments and finite element method.

The small difference that can be observed in the normal magnetic flux densities along the $x$-direction (radial direction), between the analytical result and the 3-D FEM simulation, is 
due to the curvature effects which are not taken into account in the proposed analytical model (Fig.7).

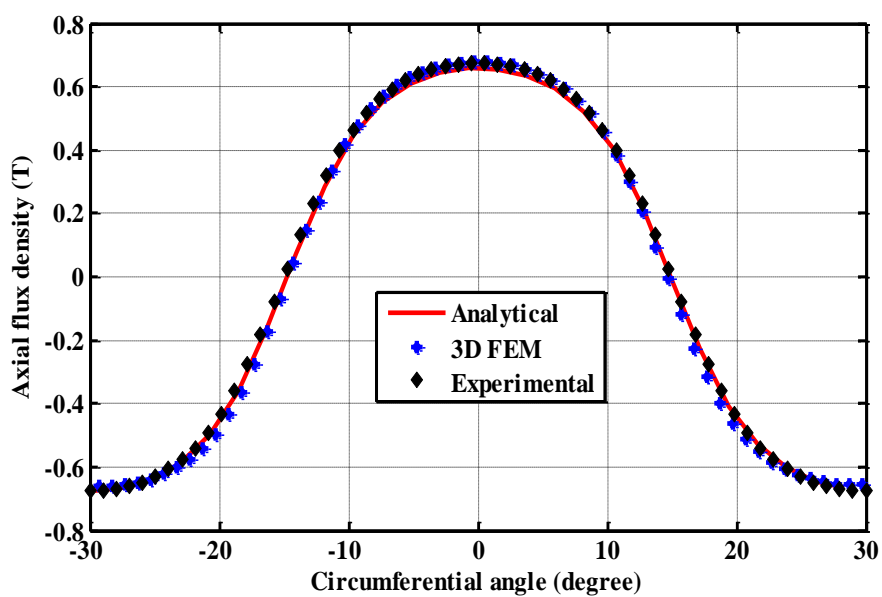

Fig.6. Axial flux density $B z$ along the angular direction in the middle of the air gap, for $e=9,5 \mathrm{~mm}$, (No-load condition).

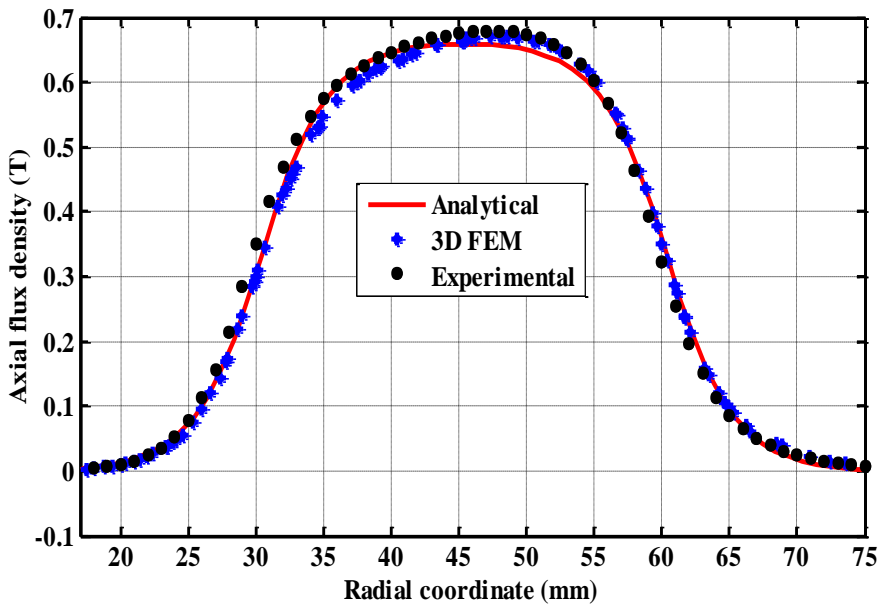

Fig.7. Axial flux density $B z$ along the radial direction in the middle of the air gap, for $e=9,5 \mathrm{~mm}$, (No-load condition).

Fig.8 shows the axial force, calculated by using the Maxwell stress tensor, as a function of the load angle. The axial force calculated with the proposed method is again in good accordance with FEM simulation.

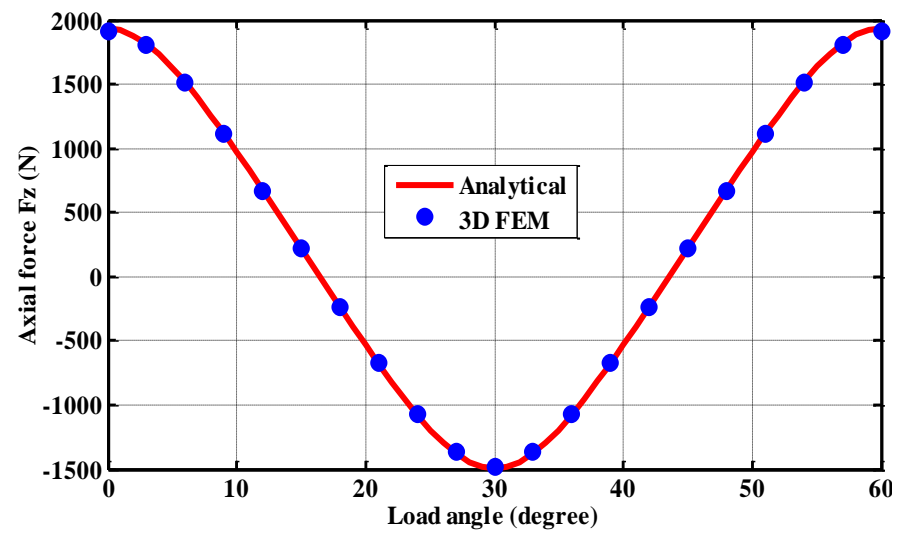

Fig.8. Axial force versus the angular displacement for $e=4 \mathrm{~mm}$.
Fig.9 and Fig.10 show the static torque variation as a function of the load angle, for two air-gap values $e=4 \mathrm{~mm}$ and $e=9.5 \mathrm{~mm}$, respectively. The static torque calculated with the proposed 3D analytical method is in very good agreement with FEM computation as well as with the measurements.

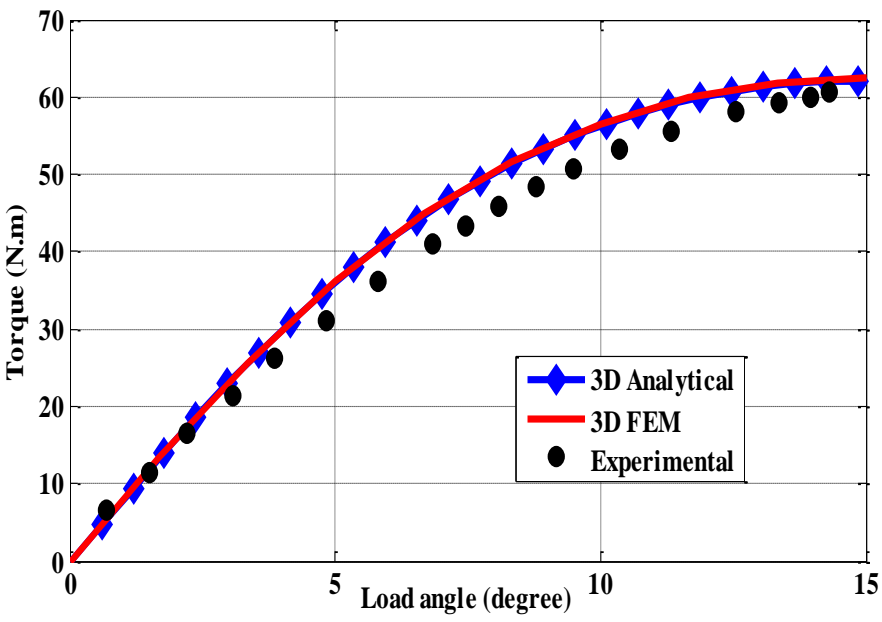

Fig.9. Torque versus the angular displacement for $e=4 \mathrm{~mm}$.

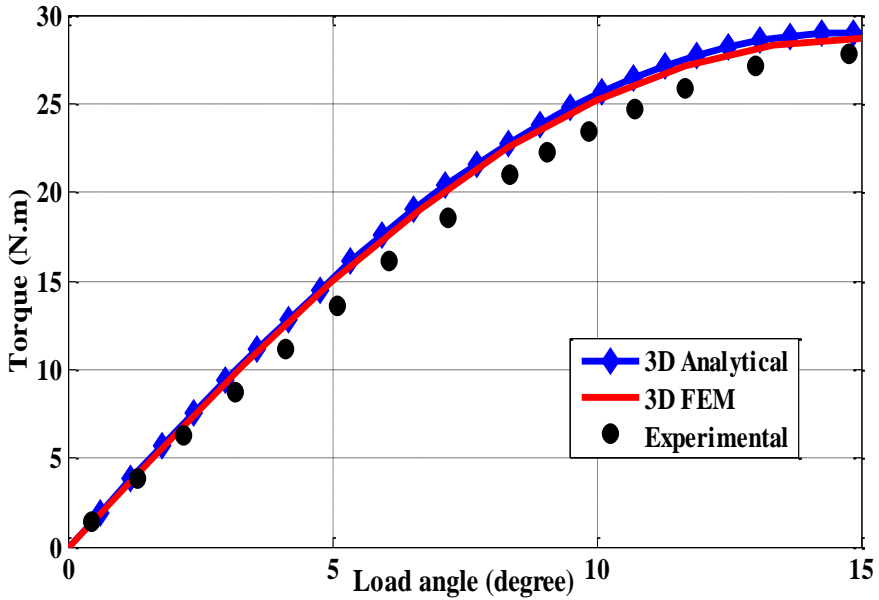

Fig.10. Torque versus the angular displacement for $e=9.5 \mathrm{~mm}$.

Knowing that a certain number of images along the $\mathrm{z}$ direction are needed to get a stable solution, the error on the torque calculation given by (16) is computed versus the number of images.

$\varepsilon_{\%}=100 \cdot \frac{\Gamma_{3 D F E M}-\Gamma_{Z}}{\Gamma_{3 D F E M}}$

Fig. 11 shows that only 2 images are needed to converge to a stable solution. So, the proposed model improves an important reduction in the computation time. The computation time (for a given position) is $24 \mathrm{~s}$ for the 3D FEM, whereas for the proposed model is about 0.014 s (on a desktop PC, 4GO, Intel core i5). 


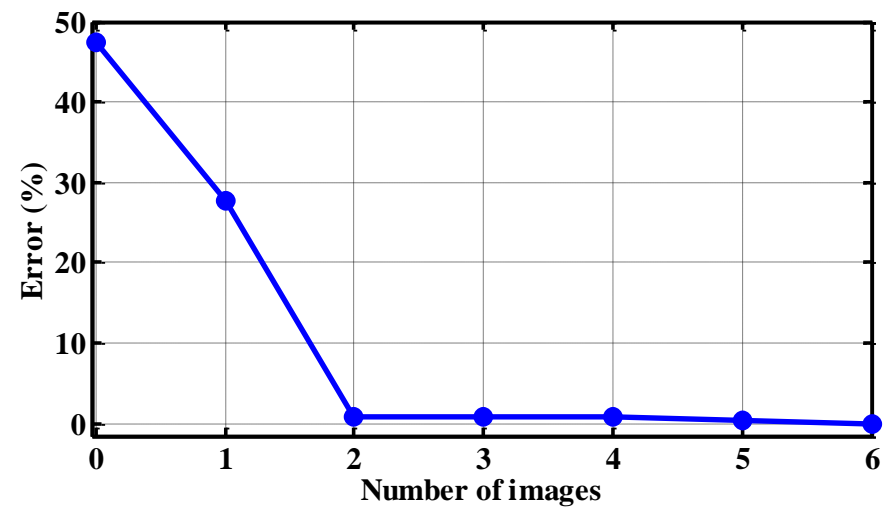

Fig.11 Error on the torque versus the numbers of images.

\section{CONCLUSION}

In this paper, a 3D analytical model, based on the equivalent magnetic charge and the theory of images is developed for calculating the forces and the torque in a PM AFMC. The analytical expressions of the force and torque are obtained using the analogy between electrostatic and magnetostatic fields, given by the Lorentz force.

The results are in good agreement to those obtained with 3D FEM and experimental with the benefit of an important reduction in the computation time. The obtained analytical expressions of forces and torque depend directly on the geometrical and physical parameters of the magnetic coupling. So, it can be used as a quick design tool. This is a very interesting advantage in any parametric or optimization study where quick models are needed.

\section{REFERENCES}

[1] M. Cong, T. Li, "Design and Application of Magnetic Coupling Used for Ultra-high Vacuum Robot, "15th International conference on mechatronics and machine vision in practice (M2VIP 08), pp346-351, 24 Dec 2008, Auckland, New-Zealand.

[2] T. Lubin, S. Mezani and A. Rezzoug, "Simple analytical expressions for the force and torque of axial magnetic couplings". IEEE Transactions on Energy Conversion, volume 27, ํㅜㄹ, pp. 536-546, 11 pages, Juin 2012.

[3] B. Dolisy, S. Mezani, T. Lubin, and J. Lévêque, "A new analytical torque formula for axial field permanent magnets coupling". IEEE Transactions on Energy Conversion, volume 30, $\mathrm{N}^{\circ} 3$, pages $892-899,8$ pages, Septembre 2015.

[4] J. Azzouzi, G. Barakat, and B. Dakyo, "Quasi-3-D analytical modeling of the magnetic field of an axial flux permanent-magnet synchronous machine," IEEE Trans. Energy Convers., vol. 20, no. 4, pp. 746-752, Dec. 2005.

[5] J. P. Yonnet, "Permanent magnet bearings and couplings," IEEE Trans. Magn., vol. 17, no. 1, pp. 1169-1173, Jan. 1981.

[6] E. Furlani, S. Reznik, and A. Kroll, "A three-dimensional field solution for radially polarized cylinders," IEEE Trans. Magn., vol. 31, no. 1, pp. 844-851, Jan. 1995.

[7] R.Wang, E. P. Furlani, and Z. J. Cendes, "Design and analysis of a permanent magnet axial coupling using 3D finite element field computations," IEEE Trans. Magn., vol. 30, no. 4, pp. 2292-2295, Jul. 1994.
[8] C. Ferreira, J. Vaudya, "Torque analysis of permanent magnet coupling using 2D and 3D finite element methods, "IEEE Trans Magnetic, 4(25):3080-3082, 2001.

[9] T. Lubin, S. Mezani, and A. Rezzoug, "Experimental and theoretical analyses of axial magnetic coupling under steady-state and transient operations," IEEE Trans. Ind. Electron., vol. 27, no. 2, pp. 4356-4365, Aug. 2014.

[10] M. Messadi, L. Hadjout, Y. Ouazir, T. Lubin, S. Mezani, A. Rezzoug, and N. Takorabet, "Eddy Current Computation in Translational Motion Conductive Plate of an Induction Heater With Consideration of Finite Length Extremity Effects," IEEE Trans.Magn,, vol. 52, Issue: 3 , 1-4, March 2016 .

[11] H. Tiegna, Y. Amara, and G. Barakat, "A new quasi-3-D analytical model of axial flux permanent magnet machines," IEEE Trans. Magn., vol. 50, no. 2, 7020204, Feb. 2014.

[12] J. P. Yonnet, S. Hemmerlin, E. Rulliere, and G. Lemarquand, "Analytical calculation of permanent magnet couplings," IEEE Trans. Magn., vol. 29, no. 6, pp. 2932-2934, Jun. 1993.

[13] R. Ravaud, G. Lemarquand,V. Lemarquand, and C.Depollier, "Permanent magnet couplings: Field and torque three-dimensional expressions based on the Coulombian model," IEEE Trans. Magn., vol. 45, no. 4, pp. 1950-1958, Apr. 2009.

[14] J.L.G. Janssen, J. J. H. Paulides, J.C. Compter, and E.A. Lomonova, "Three-Dimensional Analytical Calculation of the Torque Between Permanent Magnets in Magnetic Bearings," IEEE Trans. Magn., vol. 46, no. 6 , pp. $1748-1751$, Jun. 2010.

[15] M. Messadi, N. Takorabet , L. Hadjout, Y. Ouazir: " Three dimensional analytic model of axial flux PM motor with images method. " ISEF 2015 - XVII International Symposium on Electromagnetic Fields in Mechatronics, Electrical and Electronic Engineering, Valencia, Spain, September 10-12, 2015.

[16] H. Allag and J.-P. Yonnet, "3-D analytical calculation of the torque and force exerted between two cuboidal magnets," IEEE Trans. Magn., vol. 45, no. 10 , pp. 3969-3972, Oct. 2009.

[17] T. Lubin, and A. Rezzoug, "3-D analytical model for axial-flux eddycurrent couplings and brakes under steady-state conditions". IEEE Transactions on Magnetics, volume 51, $\mathrm{N}^{\circ} 10,8203712,12$ pages, Octobre 2015.

[18] J.M.M.Rovers, J.W.Jansen and O.A.Lomonova, "Modeling of relative permeability of permanent magnet materiel using magnetic surface charges." IEEE Trans.Magn., vol.49, no. 6, pp.2913-2919, June 2013.

[19] A. Parviainen, M. Niemelä, and J. Pyrhönen, "Modeling of axial flux permanent-magnet machines," IEEE Trans. Ind. Applications, vol. 40, no. 5, pp. 1333-1340, Sept./Oct. 2004. 